\title{
Violencia a través de las TIC: comportamientos diferenciados por género
}

\section{(Violence through ICT: behaviors differentiated by gender)}

\author{
José Domínguez Alonso \\ Universidade de Vigo(España) \\ Iago Portela Pino \\ Universidad Isabel I (España)
}

DOI: http://dx.doi.org/10.5944/ried.23.2.25916

\section{Cómo referenciar este artículo:}

Domínguez Alonso, J., y Portela Pino, I. (2020).Violencia a través de las TIC: comportamientos diferenciados por género. RIED. Revista Iberoamericana de Educación a Distancia, 23(1), pp. 273-286. http://dx.doi.org/10.5944/ ried.23.2.25916

\section{Resumen}

El impacto que tienen los estereotipos de género en la violencia a través de las tecnologías de la información y la comunicación (TIC) es una frecuente preocupación en el marco escolar, familiar e individual. El objetivo de este estudio fue conocer la influencia del género en la violencia a través de las TIC, e identificar qué variables socio-escolares están asociadas. Se ha llevada a cabo una investigación cuantitativa de tipo descriptivo-inferencial-correlacional, con una muestra de 1.495 individuos de edades entre los 10 y 17 años $(M=12.69$; DT =1.92). Se utilizó un cuestionario ad hoc (datos socio-escolares) y la escala de violencia escolar (CUVE-R) propuesta por Álvarez-García, Núñez, Rodríguez, Álvarez y Dobarro (2011). Los datos arrojan un nivel bajo-medio de violencia TIC, con mayor prevalencia en ambos géneros del envío de mensajes de ofensa, insulto o amenaza. No obstante, el género femenino lo hace a través de Internet (Tuenti, Facebook, etc.) y el masculino utilizando el móvil. Asimismo, se presentan diferencias estadísticamente significativas en el género cuando se graba o hace fotos con el móvil para hacer burlas entre compañeros o al profesorado, con mayor incidencia por parte del género masculino. También se presentan diferencias en género según la edad. La violencia TIC se estanca a partir de los 13 años en el género femenino, mientras en el masculino sigue en ligero incremento. En conclusión, el género es una variable a tener muy presente en los programas preventivos o de intervención enfocados a minimizar esta problemática.

Palabras clave: violencia; tecnologías de la información y comunicación; género; infancia; adolescencia. 


\begin{abstract}
The impact that gender stereotypes have on violence through information and communication technologies (ICT) is a frequent concern in the school, family and individual framework. The objective of the study was to know the influence of gender on violence through ICT, and identify which socio-school variables are associated. Quantitative research of descriptive-inferential-correlational type, with a sample of 1.495 individuals between the ages of 10 and $17(\mathrm{M}=12.69$; DT =1.92). An ad hoc questionnaire (socio-school data) and the scale of school violence (CUVE-R) by Álvarez-García, Núñez, Rodríguez, Álvarez and Dobarro (2011) have been used. The data show a low-medium level of ICT violence, with a higher prevalence in both genders of sending messages of offense, insult or threat. However, the female gender does it through the Internet (Tuenti, Facebook, etc.) and the male using the mobile. Likewise, there are statistically significant differences in gender when recording or taking photos with the mobile phone to make fun of peers or teachers, with a greater incidence by the male gender. There are also differences in gender according to age. ICT violence stagnates from the age of 13 on the female gender, while the male continues to increase slightly. In conclusion, gender is a variable to keep in mind in preventive or intervention programs focused on minimizing this problem.
\end{abstract}

Keywords: violence; information and communication technologies; gender; childhood; adolescence.

La violencia a través de las tecnologías de la información y la comunicación (TIC) se está generalizando entre la población más joven, con un mayor impacto en el género femenino. En efecto, dicha violencia se ha instaurado sobre todo en el periodo adolescente de manera rápida y masiva (Kowalski, Giumetti, Schroeder y Lattanner, 2014; Garaigordobil y Martínez-Valderrey, 2016), apuntando a nuevas formas de relación social entre los individuos, sobre todo, debido a un aumento en el tiempo de uso de estos dispositivos digitales (Casas, Del Rey y Ortega-Ruiz, 2013). De hecho, ningún estudio duda del uso de las redes sociales desde edades muy tempranas a través del teléfono móvil o Internet, constatando deficiencias en su uso seguro y un notable potencial de incremento en las conductas de riesgo (Garaigordobil y Aliri, 2013; Durán y Martínez, 2015; Castillejos, Torres y Lagunes, 2016; Tejada, Castaño y Romero, 2019).

Así pues, la violencia a través de las TIC engloba acciones de dominación, discriminación o abuso de posición de poder que pueden ser ejercidas por ambos géneros y acarrean riesgos a nivel psicológico, físico y social del individuo (OrtegaBarón, Buelga y Cava, 2016). Por esta razón, se encuentran investigaciones que no alcanzan diferencias de género en la violencia a través de las TIC (Hensler-McGinnis, 2008; Wall, 2008), y estudios que sostienen que el género femenino sufre más este tipo de violencia que el masculino (Reyns, 2010; Taylor, Fritsch, Liederbach y Holt, 2010). De modo paralelo, hay estudios que defienden la presencia de los hombres en conductas con mayor riesgo de ciberagresión (Li, 2006), mientras las mujeres 
lo hacen como cibervíctimas (Schneider, O'Donnell, Stueve y Coulter, 2012). No obstante, Muñiz, Cuesta, Monreal y Povedano (2015) indican que se debería tener presente los medios por los cuales se produce esta violencia mediante las TIC, para poder establecer una mayor o menor afectación en el género. También es destacable la percepción de su utilidad, mientras las chicas perciben las TIC desde una perspectiva práctica y utilitaria, los chicos las asocian a aspectos más lúdicos y de ocio (Instituto de la Mujer, 2008).

Las principales características de la violencia a través de las TIC, a saber, facilidad del anonimato del agresor, maltrato continuo en cualquier lugar, no ver las consecuencias de la conducta en la víctima por parte del agresor, pueden afectar tanto al género femenino como masculino (Serrano-Barquín y Ruiz, 2013). Sin embargo, aunque la Ley Orgánica 3/2007 de la igualdad efectiva de mujeres y hombres sustente la igualdad de oportunidades para ambos géneros en todos los ámbitos de la vida incluido el tecnológico, la brecha social en el campo de las redes sociales continúa existiendo; siendo desfavorable a las mujeres, sobre todo, en el manejo, uso y creación de contenidos (Navarro, 2009; Barragán y Ruiz, 2013).

La bibliografía actual señala una amplia variedad de factores que configuran la violencia a través de las TIC, que abarcan desde el grupo de referencia del alumnado hasta la importancia del entorno social, pasando por estudios enfocados a desarrollar competencias emocionales para la resolución de conflictos interpersonales (De la Fuente, Peralta y Sánchez, 2009; Castro-Bermúdez, 2014) o a evaluar el clima de convivencia escolar (Garretón, 2013; López de Mesa, Carvajal, Soto y Urrea, 2013; Arcila, 2014; Bengoa, 2015). No obstante, los trabajos sobre la violencia a través de las TIC suelen ignorar los aspectos de género que llevan inmersos (Tajahuerce, Franco y Juárez, 2018), siendo éste un factor relevante en sus relaciones, amistades y conflictos en la edad adolescente (Ging y O`Higgings, 2016). Algunos estudios (Slonje y Smith, 2008; Sourander, Brunstein-Klomek, Ikonen, Lindroos, Luntamo y Koskelainen, 2010; Del Río, Sábada y Bringué, 2010) sostienen que el género femenino sufre mayor acoso digital, mientras el masculino manifiesta en mayor medida hacer uso del mismo. Asimismo, Fascendini y Fialová (2011) indican que las formas de violencia relacionadas con las TIC que afectan al género femenino se centran en el hostigamiento en línea y ciberacoso, violencia por la pareja íntima, agresión sexual y violación, grabación y distribución de imágenes sexuales, avisos o mensajes falsos en internet, violencia dirigida a grupos o comunidades por identidad sexual, y casos de suicidio de mujeres jóvenes.

Al margen del debate sobre la frecuencia y gravedad de la violencia a través de las TIC, estos comportamientos violentos están presentes en todas las instituciones educativas (Landazábal y Ramírez, 2015). Cabe destacar también que los cursos de primero y segundo de educación secundaria obligatoria son los de mayor concentración de agresiones a través de las tecnologías de la información y comunicación (Calvete, Orue, Estévez, Villardón y Padilla, 2010). 
Así pues, aunque son muchas las manifestaciones de violencia que pueden surgir en contextos socio-escolares, las últimas investigaciones en este campo son unánimes al indicar una emergente violencia llevada a cabo a través de las TIC (Buelga, Cava y Musitu, 2010; Garaigordobil, Martínez-Valderrey y Machimbarrena, 2017). Esta es la principal razón por la cual, aunque en este trabajo se ha evaluado otro tipo de violencia (ejercida por el profesorado, violencia física directa o indirecta, exclusión social o disrupción), solamente se ha tenido en consideración la ejercida por el alumnado a través de las TIC.

En consecuencia, la finalidad de este estudio es evaluar el efecto del género en la violencia a través de las TIC de la población más joven. Así, el principal objetivo es descubrir y constatar, mediante la opinión del alumnado de Educación Primaria y Secundaria, la incidencia de la violencia a través de las TIC según el género e identificar qué variables socio-escolares están asociadas. De aquí surgen estas hipótesis de investigación: $\mathrm{H}_{1}$ Se espera obtener datos que confirmen una mayor presencia de violencia a través de las TIC en el género masculino. $\mathrm{H}_{2}$. Se pronostica que las diferentes conductas violentas a través de las TIC varían en función del género. Para ello, se llevó a cabo una investigación empírica, descriptiva, inferencial y comparativa (Ato, López y Benavente, 2013).

\section{MÉTODOS}

\section{Participantes}

Se ha trabajado con una muestra no probabilística e intencional, compuesta por 1.495 individuos de centros públicos (78\%) y concertados (22\%), ubicados en zonas rurales (44\%) y urbanas (56\%). Un 49.9\% de los individuos encuestados son mujeres y un $50.1 \%$ hombres. Sus edades oscilaron entre 10 y 17 años de edad $(M=12.69$; $\mathrm{DT}=1.92)$.

\section{Instrumentos}

Para la realización de este estudio se utilizó el cuestionario CUVE-R (ÁlvarezGarcía et al., 2011), que permite analizar la frecuencia de aparición de diferentes tipos de violencia escolar, protagonizado por el alumnado o el profesorado en clases. Aunque es un cuestionario enfocado al alumnado de educación secundaria, se envió también al alumnado de los dos últimos cursos de primaria (previa consulta con los autores del mismo). No obstante, solamente se han tenido en cuenta aquellos enunciados que se refieren a comportamientos violentos a través de medios electrónicos, principalmente el teléfono móvil e Internet. En concreto, se analizaron los ítems: $\mathrm{CUVE}_{6}$ (Algunos estudiantes graban o hacen fotos a compañeros o compañeras con el móvil para burlarse), $\mathrm{CUVE}_{13}$ (Ciertos estudiantes envían a 
compañeros mensajes con el móvil de ofensa, insulto o amenaza), $\mathrm{CUVE}_{20}$ (Hay estudiantes que graban o hacen fotos a profesores con móvil para burlarse de ellos), CUVE $_{24}$ (Algunos estudiantes envían mensajes a compañeros a través de las redes sociales), $\mathrm{CUVE}_{28}$ (Los estudiantes publican en Internet fotos o videos ofensivos de compañeros) y CUVE ${ }_{31}$ (Los estudiantes publican en Internet fotos o vídeos ofensivos de profesores o profesoras). Dichos ítems están redactados en forma de afirmaciones que se valoran con una escala de tipo Likert $(1=$ Nunca; 2 = Pocas veces; $3=$ Algunas veces; 4 = Muchas veces; $5=$ Siempre). La fiabilidad del cuestionario CUVE-R (factor VTIC) en este estudio es buena (Alfa de Cronbach $=.857$ ).

\section{Procedimiento}

El cuestionario se administró de manera colectiva en estudiantes de educación primaria y secundaria obligatoria de la Comunidad Autónoma de Galicia, en horario regular de clases. Después de comunicar las instrucciones oportunas y previo consentimiento informado (centro y familias), todo el alumnado cumplimentó de forma voluntaria la información solicitada. Las instrucciones explicativas fueron las mismas en todas las aulas y por el mismo encuestador, con el objetivo de evitar un factor de sesgo. Todo ello bajo las normas éticas de la Declaración de Helsinki, 1961, (revisada en Tokio en 1989 y Edimburgo en 2000), y aprobada por la Comisión de Ética de la Universidad de Vigo (España).

\section{Análisis de datos}

Todos los datos se filtraron, depuraron y fueron analizados en el paquete estadístico de SPSS v. 23.o para Windows. En primer lugar, se evaluó la confiabilidad del cuestionario utilizado a través del análisis de su consistencia interna por medio del cálculo del coeficiente del Alpha de Cronbach (un Alpha igual o mayor a 0.70 es considerado como aceptable -George y Mallery, 2003-). En segundo lugar, se calcularon porcentajes, puntuaciones medias y desviaciones típicas de los ítems. En tercer lugar, las pruebas de normalidad (Kolmogorov-Smirnov) indicaron distribuciones normales por lo cual se utilizaron las pruebas paramétricas (t-independiente). También se calculó el tamaño del efecto (d de Cohen): valores entre . 2 y .3 indican un efecto pequeño, alrededor de .5 un efecto mediano y mayores que .8 un efecto alto. Finalmente, para comprobar el grado de variación conjunta existente entre dos o más ítems, se llevó a cabo la correlación de Pearson. Para todos los análisis se considera que una relación es estadísticamente significativa cuando $\mathrm{p}$ $\leq .05$. 


\section{RESULTADOS}

A nivel general (tabla 1), la violencia ejercida a través de las tecnologías de la información y la comunicación se sitúa en unos patrones caracterizados por un nivel medio-bajo ( $\mathrm{M}=1.44$; mínimo 1 , máximo 4). De manera más específica, los individuos realizan en mayor medida el envío de mensajes de ofensa, insulto o amenaza a sus compañeros con el móvil $(\mathrm{M}=1.60)$ o a través de redes sociales $(\mathrm{M}=$ 1.52). En un nivel medio se sitúan acciones como grabar o hacer fotos a compañeros con el móvil para burlarse $(\mathrm{M}=1.44)$ y publicar en Internet fotos o videos ofensivos de compañeros $(M=1.39)$. En menor medida se sitúan los actos hacia el profesorado como grabar o hacer fotos con el móvil para burlarse $(\mathrm{M}=1.38)$ y publicar en Internet fotos o videos ofensivos $(M=1.28)$. Con respecto al género, las chicas presentan mayores medias en el envío de mensajes a compañeros a través de redes sociales de ofensa, insulto o amenaza $(M=1.54)$, mientras los chicos lo hacen en grabar o hacer fotos a compañeros con el móvil para burlarse $(\mathrm{M}=1.48)$, enviar mensajes de ofensa, insulto o amenaza a compañeros $(\mathrm{M}=1.61)$, publicar fotos o vídeos en Internet ofensivos de compañeros $(\mathrm{M}=1.42)$, grabar o hacer fotos al profesorado con el móvil para burlarse de ellos $(\mathrm{M}=1.39)$, y publicar en Internet fotos o vídeos ofensivos del profesorado ( $\mathrm{M}=1.31)$.

Seguidamente, tras el test de normalidad (Kolmogorov-Smirnov) se realizó la comparación entre las medias por la prueba $t$ de Student. En el caso de los ítems grabar o hacer fotos con el móvil entre compañeros para burlarse de ellos $(t=-2.83$, $g l=1493, p<.05)$ y grabar o hacer fotos al profesorado con el móvil con ánimo de burla $(t=-2.31, g l=1493, p<.05)$, se obtuvo diferencia significativa en el género. Así pues, el género masculino mostró una mayor presencia en el uso del móvil para grabar o hacer fotos a compañeros o profesorado que el femenino. El tamaño del efecto de la diferencia fue considerado muy bajo en grabar y hacer fotos para burlarse entre compañeros $(d=.092)$ y del profesorado $(d=.024)$. Finalmente, no se produjeron diferencias significativas en los ítems: enviar mensajes de ofensa o insulto con el móvil entre compañeros $(t=-.16, g l=1493, p>.05)$, enviar mensajes a compañeros a través de redes sociales de ofensa o amenaza $(t=-.55, g l=1493, p>.05)$, publicar en Internet fotos o vídeos ofensivos de compañeros $(t=.51, g l=1493, p>.05)$ y publicar en Internet fotos o vídeos ofensivos del profesorado $(t=-.88, g l=1493, p>.05)$. 
Tabla 1. Análisis descriptivos y diferencias entre género masculino y femenino sobre la opinión en la violencia ejercida a través de las tecnologías de la información y comunicación

\begin{tabular}{|c|c|c|c|c|c|c|c|c|c|}
\hline \multirow{2}{*}{$\begin{array}{c}\text { VIOLENCIA A TRAVÉS } \\
\text { DE LAS TIC }\end{array}$} & \multicolumn{2}{|c|}{$\begin{array}{c}\text { Total } \\
(\mathrm{N}=1495)\end{array}$} & \multicolumn{2}{|c|}{$\begin{array}{l}\text { Femenino } \\
(\mathrm{N}=746)\end{array}$} & \multicolumn{2}{|c|}{$\begin{array}{l}\text { Masculino } \\
(\mathrm{N}=749)\end{array}$} & \multirow[t]{2}{*}{$t$} & \multirow[t]{2}{*}{$p$} & \multirow[t]{2}{*}{$d$} \\
\hline & $\mathrm{M}$ & DT & M & DT & M & DT & & & \\
\hline $\begin{array}{l}\text { Algunos estudiantes } \\
\text { graban o hacen fotos a } \\
\text { compañeros o compañeras } \\
\text { con el móvil para burlarse } \\
\text { (GMC) }\end{array}$ & 1.44 & .86 & 1.40 & .81 & 1.48 & .91 & -2.83 & .046 & .092 \\
\hline $\begin{array}{l}\text { Ciertos estudiantes } \\
\text { envían a compañeros o } \\
\text { compañeras mensajes } \\
\text { con el móvil de ofensa, } \\
\text { insulto o amenaza (EMOI) }\end{array}$ & 1.60 & .99 & 1.59 & .97 & 1.61 & .98 & -.16 & .873 & - \\
\hline $\begin{array}{l}\text { Algunos estudiantes } \\
\text { envían mensajes a } \\
\text { compañeros o compañeras } \\
\text { a través de las redes } \\
\text { sociales (Tuenti, Facebook, } \\
\text { etc.) de ofensa, } \\
\text { insulto o amenaza (EMRS) }\end{array}$ & 1.52 & .93 & 1.54 & .92 & 1.51 & .94 & -.55 & .577 & - \\
\hline $\begin{array}{l}\text { Los estudiantes publican } \\
\text { en Internet fotos o vídeos } \\
\text { ofensivos de } \\
\text { compañeros o compañeras } \\
\text { (PIFV) }\end{array}$ & 1.39 & .86 & 1.36 & .81 & 1.42 & .90 & .51 & .606 & - \\
\hline $\begin{array}{l}\text { Hay estudiantes que } \\
\text { graban o hacen fotos a } \\
\text { profesores con el } \\
\text { móvil para burlarse de } \\
\text { ellos (GFP) }\end{array}$ & 1.38 & .82 & 1.37 & .80 & 1.39 & .85 & -2.31 & .048 & .024 \\
\hline $\begin{array}{l}\text { Los estudiantes publican } \\
\text { en Internet fotos o vídeos } \\
\text { ofensivos de } \\
\text { profesores o profesoras } \\
\text { (PIFVP) }\end{array}$ & 1.28 & .76 & 1.29 & .75 & 1.31 & .78 & -.88 & .378 & - \\
\hline
\end{tabular}

Nota. M: media, DT: Desviación Típica, N: número de individuos, $t$ : prueba t-independiente, $p$ : probabilidad, $d$ : tamaño de efecto.

A continuación, teniendo en cuenta la violencia a través de las TIC, el género y la edad de los individuos, se observa una mayor violencia mediante las TIC a medida que se incrementa la edad tanto para el género femenino como masculino (figura 1). No obstante, en el género femenino se mantiene el porcentaje a partir de los 11-13 
años, mientras en el masculino continúa en ligero ascenso (incremento de un 1.9\%). También se aprecia una mayor violencia TIC en los hombres menores de 11 años y mayores de 13 años. Sin embargo, entre 11 y 13 años se presenta una mayor violencia TIC en las mujeres (ligero incremento de un 1.7\%).

Figura 1. Distribución de la VTIC según el género y la edad

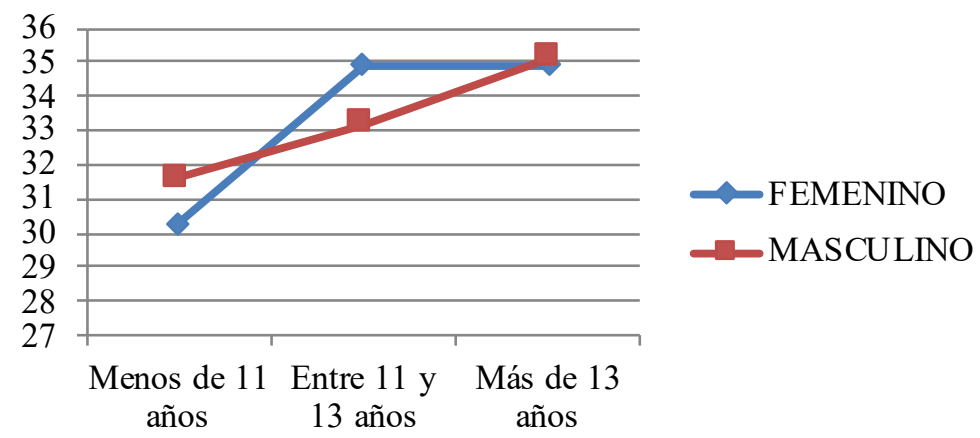

Finalmente, el estudio de correlación entre las puntuaciones de los ítems, que conforman la violencia llevada a cabo a través de las nuevas tecnologías de la información y la comunicación (tabla 2), fueron positivas, medio-altas ( $\mathrm{r}$ entre .320 y .730), y estadísticamente significativas a nivel de .001. No obstante, se muestra una mejor correlación de los ítems en el género femenino ( $r$ entre .413 y .730) que en el masculino ( $r$ entre .320 y .665).

Tabla 2. Correlaciones separadas para el género femenino y masculino en la violencia a través de las TIC

\begin{tabular}{|c|c|c|c|c|c|c|}
\hline VIOLENCIA TIC & GFM & EMOI & EMRS & PIFV & GFP & PIFVP \\
\hline GFM & - & $.465^{* *}$ & $.490^{* *}$ & $.468^{* *}$ & $.464^{* *}$ & $.413^{* *}$ \\
\hline EMOI & $.502^{* *}$ & - & $.425^{* *}$ & $.730^{* *}$ & $.514^{* *}$ & $.416^{* *}$ \\
\hline EMRS & $.482^{* *}$ & $.400^{* *}$ & - & $.454^{* *}$ & $.502^{* *}$ & $.623^{* *}$ \\
\hline PIFV & $.512^{* *}$ & $.665^{* *}$ & $.493^{* *}$ & - & $.590^{* *}$ & $.458^{* *}$ \\
\hline GFP & $.543^{* *}$ & $.56^{* *}$ & $.503^{* *}$ & $.608^{* *}$ & - & $.580^{* *}$ \\
\hline PIFVP & $.320^{* *}$ & $.374^{* *}$ & $.502^{* *}$ & $.463^{* *}$ & $.537^{* *}$ & - \\
\hline
\end{tabular}

Nota. Las correlaciones por encima de la diagonal pertenecen a la muestra de mujeres $(\mathrm{N}=$ 746), aquellas por debajo de la diagonal pertenecen a la muestra de hombres ( $\mathrm{N}=749)$. GFM: Grabar o hacer fotos con el móvil para burlarse; EMOI: Enviar mensajes de ofensa o insulto con el móvil; EMRS: Enviar mensajes de ofensa o insulto a través de las redes sociales; PIFV: Publicar en internet fotos o videos ofensivos; GFP: Grabar o hacer fotos al profesorado para burlarse; PIFVP: Publicar en Internet fotos o videos ofensivos del profesorado. Correlación significativa a los niveles ${ }^{* *} \mathrm{p}<.01$ 


\section{DISCUSIÓN}

El propósito del estudio fue descubrir y constatar, mediante la opinión del alumnado de Educación Primaria y Secundaria, la incidencia del género en la violencia a través de las TIC e identificar qué variables socio-escolares están asociadas. Los objetivos de la investigación fueron cubiertos en su totalidad, aun cuando la segunda hipótesis de investigación se cumplió parcialmente, puesto que, en la violencia ejercida mediante las TIC, el género presentó asociación con algunas de las variables evaluadas, pero no con todas.

En primer lugar, como paso previo al análisis de datos, se comprueba que el instrumento de medida utilizado es fiable. Así, en este estudio el cuestionario de violencia escolar CUVE-R presenta un buen coeficiente de fiabilidad (Alfa de Cronbach $=.886$ ) para este factor (violencia a través de las TIC). Estos valores están en la línea del trabajo realizado por Álvarez-García et al. (2011). Asimismo, resulta interesante indicar que el alumnado participante en esta investigación muestra un nivel de violencia a través de las TIC medio-bajo, con una mayor presencia de mensajes de ofensa, insulto o amenaza con el móvil o en redes sociales a compañeros.

A continuación, cabe indicar que los resultados obtenidos evidencian que se confirma la primera hipótesis de partida $\left(\mathrm{H}_{1}\right.$ Se espera obtener datos que confirmen una mayor presencia de violencia a través de las TIC en el género masculino). Aunque, ligeramente, el género masculino evidencia una mayor presencia en la violencia mediante las TIC (diferencia de medias $=+.03$ ). También difieren en la acción de violencia a través de las TIC de mayor recurrencia. El género femenino se inclina por los mensajes de ofensa, insulto o amenaza a través de las redes sociales a compañeros, mientras el género masculino prefiere grabar, hacer fotos, o enviar mensajes a compañeros con el móvil para burlarse, insultar o amenazar. Aunque no establecen diferencias según el género, estos resultados están en la línea de algunos autores relevantes (Álvarez-García, Núñez, Álvarez, Dobarro, Rodríguez y GonzálezCastro, 2011; Varela, 2012; o Álvarez-Idarriaga, 2015) que señalan como principales acciones en la violencia TIC los insultos, amenazas, burlas, suplantación de identidad o difusión de imágenes y mensajes.

Sin embargo, cabe señalar que la segunda hipótesis $\left(\mathrm{H}_{2}\right.$. Se pronostica que las diferentes conductas violentas a través de las TIC varían en función del género) se cumple de forma parcial, ya que las diferencias solamente resultaron significativas cuando la violencia se llevaba a cabo grabando o haciendo fotos a compañeros o al profesorado para burlarse con predominio del género masculino sobre el femenino. Dichos resultados sustentan investigaciones como la realizada por Baldry, Farrington y Sorrentino (2016) que indican una prevalencia similar de cibervictimización en chicos y en chicas, pero también están en la línea de algunas investigaciones (Calvete et al., 2010; Félix, Soriano, Godoy y Sancho, 2010; Álvarez-García et al., 2011) que sostienen diferencias en la violencia TIC en relación al género. Sin embargo, contradice investigaciones que señalan una mayor cibervictimización en las mujeres 
que en los hombres (Kowalsdi et al., 2014; Palermiti, Servidio, Bartolo y Costabile, 2017; Rey, Quintana-Orts, Mérida-López y Extremera, 2019).

Del mismo modo, a medida que se incrementa la edad del individuo, se eleva la presencia de violencia TIC en ambos géneros. No obstante, se mantiene estable en el género femenino a partir del tramo de edad que oscila entre los 10 y 13 años. Asimismo, se aprecia una menor incidencia de la violencia TIC en el género femenino en los individuos menores de 11 años. Algunos estudios (Garmendia, Jiménez y Mascheroni, 2017; Ramos-Soler, López-Sánchez y Torrecillas-Lacave, 2018) incorporan un nuevo perfil de usuario de las TIC forjado desde la infancia y consolidado a partir de los 10 años, que se intensifica en el periodo adolescente con mayor uso por parte del género masculino en la utilización de Internet para jugar con videoconsolas o descargar películas. Igualmente, Rodríguez-Gómez, Castro y Meneses (2018) indican que los chicos de mayor edad tienen más probabilidad de incurrir en el uso problemático en el ámbito personal y escolar de las TIC.

Finalmente, la asociación entre las acciones, que conforman la violencia a través de las TIC desde el punto de vista del alumnado, fueron positivas, oscilando de moderadas a altas, con mayores valores en el género femenino. En conclusión, los datos ponen de manifiesto la necesidad de no obviar la perspectiva de género en la violencia TIC, con la única pretensión de garantizar el derecho de las mujeres al control de las tecnologías para su libertad de movimiento y comunicación. La desigualdad en el acceso a las TIC modifica el desarrollo individual y social del individuo que afecta a su proceso de enseñanza-aprendizaje. Así pues, parece evidente que los desajustes en el acceso y utilización de las TIC hacen considerar imprescindible la incorporación de nuevas estrategias que ayuden a una mejor integración de las TIC en los jóvenes (Fisher, Boland y Lyyitinen, 2016), haciendo especial hincapié en el género femenino.

\section{REFERENCIAS}

Álvarez-García, D., Núñez, J., Rodríguez, C., Álvarez, L., y Dobarro, A. (2011). Propiedades psicométricas del Cuestionario de Violencia EscolarRevisado (CUVE-R). Revista de Psicodidáctica, 16(1), 59-83.

Álvarez-Idarriaga, G. (2015). Intervención con adolescentes victimas de ciberbullying: un abordaje desde el Trabajo Social. Revista Trabajo Social Hoy, 74, 75-91. https://doi.org/10.12960/ TSH.2015.0005

Arcila, L. (2014). Construcción de la democracia escolar y social desde los procesos de convivencia en las aulas.
Tesis de maestría. Universidad de Manizales. Manizales.

Ato, M., López, J. J., y Benavente, A. (2013). Un sistema de clasificación de los diseños de investigación en psicología. Anales de Psicología, 29(3), 1038-1059. https:// dx.doi.org/10.6018/analesps.29.3.178511

Baldry, A. C., Farrington, D. P., y Sorrentino, A. (2016). Cyberbullying in youth. Apattern of disruptive behaviour. Psicología Educativa, 22, 19-26. https:// doi.org/10.1016/j.pse.2016.02.001

Barragán, R., y Ruiz, E. (2013). Brecha de género e inclusión digital. El potencial de las redes sociales en Educación. Revista 
Profesorado: Revista de Currículum y Formación del Profesorado, 17(1), 309323.

Bengoa, J. (2015). Mejora de la convivencia escolar. La realidad nacional e internacional en los inicios del siglo XXI. Revista Mexicana de Orientación Educativa, 12(28), 14-20.

Buelga, S., Cava, M. J., y Musitu, G. (2010). Cyberbullying: victimización entre adolescentes a través del teléfono móvil y de Internet. Psicothema, 22(4), 784-789.

Calvete, E., Orue, I., Estévez, A., Villardón, L., y Padilla, P. (2010). Cyberbullying in adolescents: Modalities and aggressors' profile. Computers in Human Behavior, 26(5), 1128-1135. http://dx.doi. org/10.1016/j.chb.2010.03.017

Casas, J.A., Del Rey, R., y Ortega-Ruiz, R. (2013). Bullying and cyberbullying: Convergent and divergent predictor variables. Computers in Human Behavior, 29, 580-587. http://dx.doi.org/10.1016/j. chb.2012.11.015

Castillejos, B., Torres, C. A., y Lagunes, A. (2016). La seguridad en las competencias digitales de los millennials. Apertura (Guadalajara, Jal.), 8(2), 54-69.

Castro-Bermúdez, C. (2014). Inteligencia emocional y violencia escolar. Tesis de maestría. Universidad Militar Nueva Granada, Colombia.

De la Fuente, J., Peralta, F., y Sánchez., M. (2009). Autorregulación personal y percepción de los comportamientos escolares desadaptativos. Psicothema, 21(4), 548-554.

Del Río, J., Sádaba, C., y Bringué, X. (2010). Menores y redes ¿sociales?: de la amistad al cyberbullying. Revista de Estudios de Juventud, 88, 115-129.

Durán, M., y Martínez, R. (2015). Ciberacoso mediante teléfono móvil e Internet en las relaciones de noviazgo entre jóvenes. Revista Comunicar, 22(44), 159-167.

Fascendini, F., y Fialová, K. (2011). Voces desde espacios digitales: violencia contra las mujeres relacionada con la tecnología. Informe de síntesis en castellano sobre la investigación. Asociación para el progreso de las comunicaciones (APC).

Félix, V., Soriano, M., Godoy, C., y Sancho, S. (2010). El ciberacoso en la enseñanza secundaria. Aula Abierta, 38(1), 47-58.

Fisher, M., Boland, R., y Lyytinen, K. (2016). Social networking as the production and consumption of a self. Information and Organization, 26(4), 131-145. https://doi. org/10.1016/j.infoandorg.2016.11.001

Garaigordobil, M., y Aliri, J. (2013). Ciberacoso «Ciberbullying» en el País Vasco: diferencias de sexo y en víctimas, agresores y observadores. Behavioral Psychology / Psicología Conductual, 21(3), 461-474.

Garaigordobil, M., y Martínez-Valderrey, V. (2016). Impacto del ciberprograma 2.0 en diferentes tipos de violencia escolar y agresividad. Fronteras en Psicología, $7, \quad 428 . \quad$ https://doi.org/10.3389/ fpsyg.2016.00428

Garaigordobil, M., Martínez-Valderrey, V., y Machimbarrena, J. M. (2017). Intervención en el bullying y cyberbullying: Evaluación del caso Martín. Revista de Psicología Clínica con Niños y Adolescentes, 4(1), 25-32.

Garmendia, M., Jiménez, E., y Mascheroni, G. (2017). Riesgos y oportunidades en Internet $y$ uso de dispositivos móviles entre menores españoles (2010-2015). Madrid: Red.es / Universidad del País Vasco.

Garretón, P. (2013). Estado de la convivencia escolar, conflictividad y su forma de abordarla en establecimientos educacionales de alta vulnerabilidad social de la provincia de Concepción de Chile. [Tesis Doctoral]. Universidad de Córdoba, España.

George, D., y Mallery, P. (2003). SPSS for Windows step by step: A simple guide and reference. 11.o update (4th ed.). Boston: Allyn \& Bacon. 
Ging, D., y O'Higgins, J. (2016). Cyberbullying, conflict management or just messing? Teenage girl's understandings and experiences of gender, friendship, and conflict on Facebook in an Irish second-level school. Feminist Media Studies, 16(5), 805-821. http://dx.doi.org/10.1080/14680777.201 5.1137959

Hensler-McGinnis, N. F. (2008). Cyberstalking victimization: Impact and coping responses in a national university sample. [Tesis doctoral], Facultad deEducación, Universidad de Maryland, Estados Unidos. https://doi.org/10.1037/ e611792009-001

Kowalski, R. M., Giumetti, G. W., Schroeder, A. N., y Lattanner, M. R. (2014). Bullying in the digital age: A critical review and meta-analysis of cyberbullying research among youth. Psychological Bulletin, 14O, 1073-1137. https://doi.org/10.1037/ $\underline{\mathrm{a} 0035618}$

Instituto de la Mujer (2008). Mujeres y nuevas tecnologías de la información y la comunicación. Madrid: Ministerio de la Igualdad. Gobierno de España.

Landazábal, M. G., y Ramírez, J. A. O. (2015). Estudios epidemiológicos sobre la incidencia del acoso escolar e implicaciones educativas. Informació Psicològica, 94, 14-35.

Li, Q. (2006). Cyberbullying in Schools: A research of gender differences. School Psychology International, 21(2), 157-170.

López de Mesa, C., Carvajal, C., Soto, M., y Urrea, P. (2013). Factores asociados a la convivencia escolar en adolescentes. Educación y Educadores, 16(3), 383-410. https://doi.org/10.5294/edu.2013.16.3.1

Muñiz, M., Cuesta, P., Monreal, M., y Povedano, A. (2015). Online teen dating violence, family and school climate from a gender perspective. Revista sobre la Infancia y la Adolescencia, 9, 85-97. http://dx.doi.org/10.4995/ reinad.2015.3898
Navarro, M. (2009). La brecha digital de género en España: cambios y persistencias. Revista Fenimismo/s, 14. Centro de estudios sobre la Mujer de la Universidad de Alicante.

Ortega-Barón, J., Buelga, S., y Cava, M. J. (2016). The influence of school climate and family climate among adolescents victims of cyberbullying. Comunicar, 24(46), 57-65. https://doi.org/10.3916/ C46-2016-06

Palermiti, A. L., Servidio, R., Bartolo, M. G., y Costabile, A. (2017). Cyberbullying and self-esteem: An Italian study. Computers in Human Behavior, 69, 136-141. https:// doi.org/10.1016/j.chb.2016.12.026

Ramos-Soler, I., López-Sánchez, C., y Torrecillas-Lacave, T. (2018). Percepción de riesgo online en jóvenes y su efecto en el comportamiento digital. Comunicar, 56(XXVI), 71-79. https://doi. org/10.3916/C56-2018-07

Rey, L., Quintana-Orts, C., Mérida-López, S., y Extremera, N. (2019). Being Bullied at School: Gratitude as Potential Protective Factor for Suicide Risk in Adolescents. Frontiers in Psychology, 10, 662. https:// doi.org/10.3389/fpsyg.2019.00662

Reyns, B. (2010). Being Pursued Online: Extent and Nature of Cyberstalking Victimization from a Lifestyle/Routine Activities Perspective. [Tesis doctoral], Facultad de Educación, Universidad de Cincinnati, Estados Unidos.

Rodríguez-Gómez, D., Castro, D., y Meneses, J. (2018). Problematic uses of ICTs among young people in their personal and school life. [Usos problemáticos de las TIC entre jóvenes en su vida personal y escolar]. Comunicar, 56, 91-100. https://doi. org/10.3916/C56-2018-09

Schneider, S. K., O’Donnell, L., Stueve, A., y Coulter, R. W. S. (2012). Cyberbullying, school bullying, and psychological distress: A regional census of high school students. American Journal of Public 
Health, 102(1), 171-177. https://doi. org/10.2105/AJPH.2011.300308

Serrano-Barquín, R., y Ruiz, E. (2013). Violencia simbólica en internet. Raximhai,9(3), 121-139. https://doi. org/10.35197/rx.09.03.e.2013.06.rs

Slonje, R., y Smith, P. K. (2008). Cyberbullying: Another main type of bullying?: Personality and social sciences. Scandinavian Journal of Psychology, 49(2), 147-154. http://dx.doi. org/10.1111/j.1467-9450.2007.00611.x

Sourander, A., Brunstein-Klomek, A., Ikonen, M., Lindroos, J., Luntamo, T., y Koskelainen, M. (2010). Psychosocial risk factors associated with cyberbullying among adolescents. A populationbased study. Archives of General Psychiatry, 67(7), 720-728. http://dx.doi. org/10.1001/archgenpsychiatry.2010.79

Tajahuerce, I., Franco, Y. G., y Juárez, J. (2018). Ciberbullying y género: nuevos referentes en la ocupación de los espacios virtuales. Estudios sobre el Mensaje Periodístico, 24(2), 1845-1859. http:// dx.doi.org/10.5209/ESMP.62250

Taylor, R. W., Fritsch, E.J.,Liederbach, J., y Holt, T. J. (2010). Digital crime and digital terrorism. Nueva Jersey: Pearson Prentice Hall.

Tejada, E., Castaño, C., y Romero, A. (2019). Los hábitos de uso en las redes sociales de los preadolescentes. RIED. Revista Iberoamericana de Educación a Distancia, 22(2), 119-133. http://dx.doi. org/10.5944/ried.22.2.23245

Varela, R. (2012): Violencia, Victimización $y$ cyberbullying en adolescentes escolarizados/as: una perspectiva desde el Trabajo Social. Tesis doctoral. Facultad de Ciencias Sociales, departamento de Trabajo Social y Servicios Sociales. Universidad Pablo de Olavide.

Wall, D. S. (2008). Cybercrime: The transformation of crime in the information age. Cambridge: PolityPress.

\section{PERFIL ACADÉMICO Y PROFESIONAL DE LOS AUTORES}

Jose Domínguez Alonso. Doctor en Psicopedagogía, Licenciado en Ciencias de la Educación y Psicopedagogía. Profesor de la Universidad de Vigo (Campus Ourense). Departamento de Análisis e Intervención Psicosocioeducativa. Área de Metodología de las Ciencias del Comportamiento. Investigación: Estudio de las metodologías cualitativa y cuantitativa aplicadas al campo de la educación: atención a la diversidad, violencia escolar, inclusión y exclusión social. Correo electrónico: E-mail: jdalonso@uvigo.es

Iago Portela Pino. Profesor Doctor en Ciencias de la Salud y del Deporte (Universidad Isabel I). Master en Condicionantes Genéticos, Nutricionales y Ambientales del Crecimiento y Desarrollo. Investigación: Estudio en el campo de la salud y el deporte: calidad de vida, motivación y abandono del ejercicio físico. E-mail: iportela@uvigo.es

Dirección:

Plaza Eduardo Barreiros, $\mathrm{n}^{\circ} 4,2^{\circ} \mathrm{b}$, 32004 - Ourense (España) 
Fecha de recepción del artículo: 21/10/2019

Fecha de aceptación del artículo: 10/12/2019

Fecha de aprobación para maquetación: 20/02/2020 